We appreciate the response to this publication feature - and welcome all contributions. Contributions may be sent to Phil Oshel, our Technical Editor at:

$$
\begin{array}{ll}
\text { Mr. Phil Oshel } & \text { (608)833-2885 } \\
\text { PO Box 620068 } & \text { Fax: (608)836-1969 } \\
\text { Middleton WI 53562 } & \text { eMail: oshel@terracom.net }
\end{array}
$$

\section{Removing Platinum/Carbon Replicas From Mica}

There are probably as many different ways to approach this kind of problem as there are people doing platinum replicas! The most reliable method we have ever discovered is to use a $1 \%$ aqueous polyacrylic acid (PAA) solution, deposited as one drop on the surface with the stubborn replica. Twenty four hours later, the drop has spread and the water has evaporated, leaving a thin but very tough PAA film. Then with something sharp (e.g., scalpel blade, but wear eye protection for this!), slide the blade edge underneath the edge of the PAA film, and if you have the "art", it will literally just pop off with the Pt/carbon film.

Next the PAA is floated on a surface of water, carbon side up, and again, do other things and come back 24 hours later. The PAA will have all dissolved into the water, leaving the Pt/carbon film floating on the surface of the water. The film is then picked up on grids as you would any other floating film.

Note: Patience of clearly a virtue, be sure to give it the full 24 hours or your grids will be PAA contaminated, with a major loss of contrast. Use a deep petri dish for this so that there is sufficient volume of water to efficiently dissolve the PAA.

If this sounds too complicated and you don't have patience, there is an alternative we also use: Victawet for EM. The Victawet is evaporated from a tungsten basket (see website instructions) and a very thin layer of the release agent is deposited onto the mica (or glass slide). Then apply the samples, shadow with $\mathrm{Pt} / \mathrm{C}$ and the replica now is almost guaranteed to float off on the first try.

One note: The "better" the grade of mica, I am told, the easier is the release of such films. Grade V1 mica supposedly releases easier and that might be because there are fewer cleavage steps. We have not tested that theory ourselves so on that there can be no guarantees, but it does make sense.

$$
\begin{gathered}
\text { Charles Garber, SPI Supplies } \\
\text { cgarber@2spi.com }
\end{gathered}
$$

\section{Dehydrating Delicate Specimens for Light Microscopy}

Dehydrating delicate or highly hydrated specimens often results in serious distortions. One method to avoid this is to put the properly fixed specimen in $10 \%$ glycerol, then leave it in an open jar with suction (e.g. a vacuum desiccator, or a vacuum oven that's not switched on) until the water has evaporated the volume will be reduced down to $10 \%$ ). Mark the level on the side of the jar when you start. Alcohol can then be added gradually to the glycerol + specimen until it's about $95 \%$ alcohol and $5 \%$ glycerol, then go into $100 \%$ alcohol. (100\% alcohol must be used, as glycerol doesn't mix with clearing agents or wax.) I've done this occasionally, and it takes a couple of weeks to evaporate the water with our building's feeble vacuum line. End results are fine.

This glycerol method for dehydrating delicate specimens is often used for bits of plant that have complex shapes but consist mostly of water. Rapid or even moderate solvent changes will make the cellulose cell walls collapse. For a detailed account of the method, Berlyn, G.P. \& J.P. Miksche, 1976. Botanical Microtechnique and Cytochemistry. lowa State Univ Press, Ames. This book, which you can buy directly from the publisher, is a nicely bound hardback, is full of useful information and is unbelievably cheap (oops! - inexpensive).

Rather surprisingly, chemical dehydration with 2,2dimethoxy propane (DMP) is also quite gentle on delicate specimens. (It was first used for scanning EM.) It's described in Berlyl \& Miksche (a pretty new method back in 1976) and there's a more recent account in Biotechn. \& Histochem. 74: 20-26 (Jan 1999). The DMP method is much faster than glycerol, needing only $10-15$ minutes for a $1 \mathrm{~mm}$ specimen, so you might want to try it first.

John A. Kiernan, The University of Western Ontario jkiernan@julian,uwo.ca

\section{SEM Specimen Drying}

CPD certainly did become the most popular method by a country mile for SEM specimen drying, but freeze-drying still has advantages over it in some situations. These include, for example, specimens where lipid content or lipid structures must be retained (e.g. plant and insect epicuticles), where the specimen is an aggregate of objects loosely bound by a fluid or a mucilaginous matrix (e.g. it could be an advantage in Tony Kowal's yeast and bacterial colonies, soils and clays), or where the specimen is mechanically fragile and the components would be dispersed on submersion in baths of liquid during fixation and solvent drying and CPD (soils \& clays, fungal sporangia, yeast and bacterial colonies).

The down side of freeze-drying in most of these contexts is that some shrinkage and distortion almost always results. Consequently, for almost all the situations listed above, and a host of others as well, Low-temperature SEM became the method of choice. In LTSEM the specimen can be viewed fully frozen-hydrated, but most commercially-available LTSEM systems have specimen temperature control, and full or partial freeze-drying can be undertaken either on the SEM specimen stage or in the cryo-preparation unit if required.

Anyone seeking a freeze-drier unit for EM specimens should contact Emitech (www.Emitech.co.uk) who make a peltier-cooled unit $(K 750)$ which operates around $-60^{\circ} \mathrm{C}$ (and is not unlike the Edwards-Pearce tissue drier) and a turbo molecular pumped Liquid nitrogen cooled low-temperature freeze drier $(\mathrm{K} 775)$ which operates $<-80^{\circ} \mathrm{C}$.

Chris Jeffree, The University of Edinburgh cjeffree@srv0.bio.ed.ac.uk

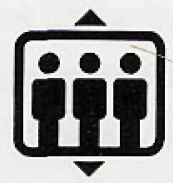

